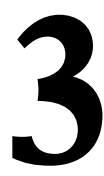

\title{
EL IMPACTO BIBLIOMÉTRICO DEL MOVIMIENTO MOOC EN LA COMUNIDAD CIENTÍFICA ESPAÑOLA
}

\section{(THE BIBLIOMETRIC IMPACT OF MOOC MOVEMENT IN THE SPANISH SCIENTIFIC COMMUNITY)}

Ignacio Aguaded Gómez

Universidad de Huelva

Esteban Vázquez-Cano

Universidad Nacional de Educación a Distancia

Eloy López-Meneses

Universidad Pablo de Olavide, Sevilla

DOI: $10.5944 / e d u c X X 1.16454$

Cómo referenciar este artículo/How to reference this article:

Aguaded Gómez, I.; Vázquez-Cano, E. y López-Meneses, E. (2016). El impacto bibliométrico del movimiento MOOC en la Comunidad Científica Española. Educación XX1, 19(2), 77-104, doi: 10.5944/educXX1. 16454

Aguaded Gómez, I.; Vázquez-Cano, E. \& López-Meneses, E. (2016). El impacto bibliométrico del movimiento MOOC en la Comunidad Científica Española. [The bibliometric impact of MOOC movement in the Spanish Scientific Community]. Educación XX1, 19(2), 77-104, doi: 10.5944/ educXX1. 16454

\section{RESUMEN}

España ha sido durante el año 2013 el país líder europeo en oferta de cursos MOOC y se encuentra en una posición puntera a nivel mundial en el número de cursos masivos ofertados en 2014. Esta prolífica actividad se está trasladando al mundo divulgativo y científico en forma de entradas en blogs, redes sociales y páginas web, así como en forma de artículos científicos y libros que intentan analizar el movimiento desde diferentes aproximaciones metodológicas. Hasta el momento, no se ha analizado la repercusión bibliométrica del impacto del movimiento MOOC en la comunidad científica española, por lo que el objetivo de esta investigación, es realizar un estudio bibliométrico de la repercusión científica en forma de artículo o libro de investigación en revistas y editoriales españolas durante el periodo comprendido entre enero de 2010 y junio de 2014. El estudio se afronta desde una metodología descriptiva y cuantitativa tomando como referencia indicadores bibliométricos de producción, en número de citas e indicadores de visibilidad y de impacto en diferentes bases de datos: Wos/Social Science Citation Index, Scopus, In-Recs, Google Scholar y la categorización de revistas científicas españolas (ANEP/ FECYT). Los resultados muestran que el impacto de la producción científica 
española en formato libro y artículo en prestigiosas bases de datos internacionales (Wos-SSCI/Scopus) es muy bajo, aunque el impacto nacional según categorización ANEP/FECYT e In-Recs es moderadamente alto.

\section{PALABRAS CLAVE}

MOOC; estudio bibliométrico; impacto; producción científica; revistas españolas.

\section{ABSTRACT}

Spain was in 2013 the leading European country in MOOC course offerings and is in a leading position worldwide in the number of massive courses offered in 2014 This prolific activity is being transferred to the educational and scientific world in the form of posts in blogs, social networks and web pages, as well as scientific papers and books that attempt to analyze the movement from different methodological approaches. To date there is not any research that analyzes the bibliometric impact of MOOC movement in the Spanish scientific community. Therefore the objective of this research is to perform a bibliometric study of the scientific impact in the form of scientific article or research book in journals and Spanish publishers from January 2010 to June 2014. The study was approached from a descriptive and quantitative methodology taking as reference bibliometric indicators of production, number of citations, and indicators of visibility according to their impact on different databases: Wos / Social Science Citation Index, Scopus, In-Recs, Google Scholar and categorization of Spanish scientific journals (ANEP / FECYT). The results show that the impact of the Spanish scientific production in form of book and scientific article in prestigious international databases (Wos-SSCI / Scopus) is very low, although the national impact categorization according to ANEP / FECYT and In-Recs is moderately high.

\section{KEY WORDS}

MOOCs; bibliometric study; impact; scientific production; Spanish journals.

\section{INTRODUCCIÓN}

El impacto de los cursos masivos, en línea y en abierto denominados con la sigla inglesa «MOOC» (Massive Open Online Course) ha sido incuestionable en estos cuatro últimos años (Cormier y Siemens, 2010; Siemens, 2012; Downes, 2012, 2013; Yuan y Powell, 2013; Dillenbourg et al., 2014). Los MOOC han irrumpido con fuerza en el contexto de la formación superior y el movimiento ya es considerado como uno de los mayores acontecimientos disruptivos en los últimos años (Pappano, 2012; Anderson, 2013; Conole, 2013; Little, 2013; Aguaded, 2013). Sin lugar a dudas, el movimiento MOOC 
ha supuesto un punto de inflexión para la Educación Superior, pero para su definitiva consolidación se precisa de un fuerte componente investigador que analice desde diferentes posturas los principios sobre los que se asienta el movimiento y, especialmente, en aquellos aspectos que presentan más controversia: el modelo pedagógico en el que se sustenta, el reto tecnológico que implica dar una respuesta adecuada a la masividad y los retos ante la certificación y la monetización que garanticen una futura sostenibilidad y su definitivo asentamiento en al panorama formativo de la Educación Superior (Eaton, 2012; Zapata, 2013; Aguaded, Vázquez-Cano y Sevillano, 2013; Guàrdia, Maina y Sangrà, 2013; Sangrà, 2013; Hoxby, 2014; DeBoer et al., 2014).

Los MOOC han acaparado este interés mundial debido a su gran potencial para ofrecer una formación gratuita, ofertada por prestigiosas universidades y accesible a cualquier persona independientemente de su país de procedencia, su formación previa y sin la necesidad de pagar por su matrícula (Daniel, 2013; Christensen et al, 2013; Vázquez-Cano, López Meneses y Sarasola, 2013; Radford et al., 2014). La evolución de la educación a distancia y los avances tecnológicos constituyen una importante oportunidad para incrementar el acceso a la educación y contribuir al cumplimiento de los compromisos educativos internacionales. Al respecto, la UNESCO (2013: 7) considera que estos procesos masivos en abierto y gratuitos son una oportunidad para proporcionar una formación en diferentes contextos y la posibilidad de garantizar a la población mundial una formación a lo largo de la vida.

España se encuentra en una posición puntera mundial en la oferta de cursos MOOC. En agosto del 2014 el portal europeo «Open Education Europa» (http://goo.gl/w0wdpo) contabilizaba 253 cursos MOOC en España; lo que representa el 34,09\% de todos los cursos MOOC ofertados por instituciones europeas. Si observamos la demanda, es decir, el volumen de participación en la oferta mundial de MOOC, volvemos a encontrar España dentro de los cinco países con más estudiantes matriculados en este tipo de formación, junto con países como EEUU, Reino Unido, Canadá o Brasil (Oliver et al., 2014).



Figura 1. Distribución de la oferta de cursos MOOC en Europa

Fuente: «Open Education Europa», 1 de agosto de 2014. 
Este contexto ha generado que la investigación en MOOC se haya multiplicado en estos últimos años y que en la actualidad haya alcanzado un auge considerable. Muestra de ello son las «llamadas a artículos» que diferentes revistas españolas de impacto han lanzado este año 2014, entre las que destacan: el monográfico número 44 en la Revista Comunicar, titulado: MOOC (Cursos masivos abiertos en línea): interactividad comunicativa y multimedia. El monográfico 18(1) publicado en la «Revista Profesorado. Revista de currículum y formación del profesorado», titulado: «MOOC y la educación superior. La expansión del conocimiento» y la «llamada a artículos» de la Revista RUSC, con un monográfico titulado: «Los MOOC: ¿una transformación radical o una moda pasajera?». Asimismo, se han publicado otras investigaciones como el «Informe Scopeo» patrocinado por la Universidad de Salamanca y titulado: «MOOC: Estado la situación actual, posibilidades, retos y futuro» (2013) y el informe titulado: «MOOC en España» de la Cátedra Telefónica (2014), entre diferentes artículos y libros diseminados en revistas y editoriales españolas.

El movimiento MOOC, al igual que cualquier ciencia o campo de estudio, requiere de procesos de recuperación, evaluación y análisis que brinden la posibilidad de visualizar y representar de forma exhaustiva, consistente, relevante y precisa el resultado de su labor y que asegure la legitimidad y originalidad del conocimiento científico producido. Hasta la fecha, no se ha realizado un estudio bibliométrico del movimiento MOOC en el panorama científico español, atendiendo al impacto de las revistas en bases de datos de reconocido prestigio y conforme a variables que permitan al investigador tener un panorama actualizado y completo del tipo de producción publicada en el ámbito español. Por este motivo, esta investigación se ha organizado para dar respuesta a los siguientes objetivos:

- Cuantificar bibliométricamente la producción científica española sobre la temática MOOC publicada en el periodo 2010-14 con respecto a las siguientes variables: número total de artículos publicados y su evolución por área de conocimiento, revistas, editoriales e instituciones con mayor índice de publicación, impacto en bases de datos, autores, número de citas recibidas, principales revistas citantes, promedio de citas por año y enfoque metodológico de los artículos.

- Proporcionar un estado de la cuestión actual y completo de la producción científica española sobre MOOC que sirva de referencia para futuros estudios e investigaciones. 


\section{MÉTODO}

Esta investigación parte de los principios enmarcados en los estudios bibliométricos en el campo de la educación (Fernández y Bueno, 1998; Ariza et al., 2011; Fernández, 2011; Gómez-García et al., 2012), con el empleo de técnicas de tipo descriptivo y cuantitativo (Alcaín y San Millán, 1993; Vanti, 2000; Peritz y Bar-Ilan, 2002; Davis y González, 2003). El uso comparado de bases de datos es un método utilizado en investigaciones que miden el impacto de un término o tendencia. Para analizar el impacto en el panorama científico español, hemos recurrido a dos tipos de indicadores bibliométricos: de producción, en número de citas e indicadores de visibilidad y de impacto conforme a su repercusión bibliométrica en diferentes bases de datos (Buela-Casal, 2010; Velasco et al., 2012).

\section{ANÁLISIS DE DATOS}

Con el mismo objetivo conceptual de medir el impacto de un término o tendencia desde una perspectiva bibliométrica, distintas disciplinas científicas han evidenciado éxito en su aplicación (Cahlik, 2000; Estabrooks et al., 2004; Chiu y Ho, 2005; Neff y Corley, 2009).

El procedimiento de análisis de datos abarca el periodo de estudio desde el 1 enero de 2010 hasta el 30 de junio de 2014 y la extracción de datos para el estudio del impacto del movimiento MOOC en la comunidad científica española se ha realizado atendiendo a las siguientes fases:

1. Utilización de la base de datos DICE (http://dice.cindoc.csic.es). Es una herramienta creada por el Grupo de Investigación Evaluación de publicaciones científicas en Ciencias Sociales y Humanas (EPUC) del Instituto de Estudios Documentales sobre Ciencia y Tecnología (IEDCYT) del Centro de Ciencias Humanas y Sociales (CCHS) del CSIC. La ANECA (Agencia Nacional de Evaluación de la Calidad y Acreditación) utiliza esta base de datos como referencia de calidad de las publicaciones españolas, en sus procesos de evaluación de profesorado. Con esta base de datos, se filtraron tres áreas temáticas para la extracción: Comunicación. Ciencias de la educación y Biblioteconomía y Documentación.

2. Selección de la categoría ANEP/FECYT. La Agencia Nacional de Evaluación y Prospectiva (ANEP), entre otras funciones, analiza y publica el impacto de las revistas españolas científicas conforme a los siguientes criterios de calidad científica: A+, A, B y C (alto-A+/A, medio-B y bajo-C). 
3. Extracción de cada una de las revistas en las que ha aparecido un artículo en el periodo desde el 1 enero de 2010 al 30 de junio de 2014, en cuyo título, en el resumen o en las palabras claves contenían los términos MOOC, MOOCs o COMA (traducción al español de las siglas MOOC).

4. Cada uno de los artículos localizados se ha clasificado descriptivamente conforme a los siguientes datos: título, ISSN, nombre de la Revista, año, categoría ANEP, Bases de datos de indexación, autores, revistas citantes, número de citas, institución a la que pertenece la revista, área de conocimiento y enfoque metodológico.

5. Las variables que se obtienen de manera directa de la revista en la que se encuentra el artículo seleccionado son: Título, ISSN, Nombre de la Revista, Año, Autores e Institución a la que pertenece la revista.

Asimismo, se han incluido las citas de los artículos previamente seleccionados en DICE, que aparecían recogidas en SSCI (WoS) y Scimago (Scopus). Se ha interrogado también la base de datos Dialnet con los términos «MOOC»y «MOOCs», lo que ha permitido incorporar los artículos de temática MOOC que no habían sido registrados previamente en la búsqueda efectuada en DICE. Los datos de indexación han sido tomados de las bases DICE y MIAR. La información de los «cuartiles» correspondiente a los artículos tratados en el estudio se ha extrapolado de consultas realizadas a la base de datos In-Recs (http://ec3.ugr.es/in-recs/). Las bases de datos utilizadas para determinar la indexación de las editoriales de libros han sido: Catálogo del ISBN, Google Books, Google Scholar y SPI (Scholarly Publishers Indicators).

Los indicadores empleados en el presente estudio han sido:

- Título.

- Carácter: Libro o artículo.

- Área de conocimientos de la revista: Educación, Comunicación, Biblioteconomía y Documentación.

- Autor/res.

- ISBN / ISSN.

- Revista / Editorial. 
- Año de publicación.

- Categoría ANEP.

- Cuartil In-Recs.

- Citas (Google Scholar).

- Citas (Wos/SSCI).

- Citas (Scopus).

- Indexación en otras bases de datos.

- Citas recibidas.

- Principales revistas citantes.

- Nombre de la institución.

- Área de conocimiento.

- Enfoque metodológico del artículo.

\section{RESULTADOS}

Los resultados se han organizado tomando como referencia las variables de la investigación, de forma que se pueda analizar la repercusión bibliométrica del movimiento MOOC conforme a criterios estandarizados de referencia en el mundo científico. Asimismo, en el anexo I se puede comprobar el listado completo de artículos y libros científicos que se han obtenido para el intervalo de tiempo y variables contempladas.

En primer lugar, presentamos la evolución de la publicación de artículos y libros según área de conocimiento entre enero de 2010 y junio de 2014 (Tabla 1). La evolución del movimiento en España por área de conocimiento se ha centrado principalmente en el área de Ciencias de la Educación que acapara el $89,5 \%$ de los artículos publicados y el $75 \%$ de los libros del total de publicaciones sobre MOOC. 
Tabla 1.

Evolución de la publicación de artículos y libros sobre MOOC (2010-2014) por área de conocimiento

Área de conocimiento $(\%)$

\begin{tabular}{lccc}
\hline Tipo de documento & Comunicación & $\begin{array}{c}\text { Ciencias } \\
\text { de la Educación }\end{array}$ & $\begin{array}{c}\text { Biblioteconomía } \\
\text { y Documentación }\end{array}$ \\
\hline Libros & 0,0 & 75,0 & 25,0 \\
Artículos de revistas & 7,9 & 89,5 & 2,6 \\
\multicolumn{1}{c}{ Total \% } & $\mathbf{7 , 1 4}$ & $\mathbf{8 8 , 1 0}$ & $\mathbf{4 , 7 6}$ \\
\hline
\end{tabular}

En el intervalo de estudio analizado, se han publicado un total de 38 libros y 4 libros. El impacto del movimiento en España se ha producido con una mayor incidencia a partir del año 2013 (11 artículos y 2 libros) y más acusadamente en los seis primeros meses del año 2014 (27 artículos y 2 libros). Como se puede comprobar, los tres primeros años no arrojan ningún resultado conforme a los criterios de impacto considerados en el estudio, según podemos comprobar en la Tabla 2.

Tabla 2.

Evolución de la publicación de artículos y libros sobre MOOC (2010-2014)

\begin{tabular}{ccc}
\hline Año de publicación & Libros & Artículos \\
\hline 2010 & 0 & 0 \\
2011 & 0 & 0 \\
2012 & 0 & 0 \\
2013 & 2 & 11 \\
2014 & 2 & 27 \\
Total & $\mathbf{4}$ & $\mathbf{3 8}$ \\
\hline
\end{tabular}

Las revistas y editoriales que se han significado con una mayor producción en temática MOOC se pueden observar en la Tabla 3. Cuatro revistas españolas acaparan el $54,75 \%$ de las publicaciones sobre MOOC (Profesorado. Revista de currículum y formación del profesorado. RED. Revista de Educación a Distancia. Digital Education Review. RIED. Revista Iberoamericana de Educación a Distancia). El 26,19\% de la Revista Profesorado está motivado por la «llamada a artículos» que se realizó para el número 18(1). 
Tabla 3.

Revistas y editoriales con mayor índice de publicación en MOOC

\begin{tabular}{|c|c|}
\hline Revista / Editor & $\%$ \\
\hline Profesorado. Revista de Currículum y Formación del Profesorado & 26,19 \\
\hline RIED. Revista Iberoamericana de Educación a Distancia & 11,90 \\
\hline Digital Education Review & 9,52 \\
\hline RED. Revista de Educación a Distancia & 7,14 \\
\hline Comunicar & 4,76 \\
\hline Revista de Educación y Derecho = Education and Law Review & 4,76 \\
\hline RUSC. Revista de Universidad y Sociedad del Conocimiento & 4,76 \\
\hline Anuario ThinkEPI & 2,38 \\
\hline @tic: Revista d’Innovació Educativa & 2,38 \\
\hline Ediciones Universidad de Salamanca & 2,38 \\
\hline $\begin{array}{l}\text { GECONTEC: Revista Internacional de Gestión del Conocimiento y } \\
\text { la Tecnología }\end{array}$ & 2,38 \\
\hline Historia y Comunicación Social & 2,38 \\
\hline IJERI: International Journal of Educational Research and Innovation & 2,38 \\
\hline Innovación Educativa & 2,38 \\
\hline Journal for Educators, Teachers and Trainers & 2,38 \\
\hline Octaedro & 2,38 \\
\hline REDU: Revista de Docencia Universitaria & 2,38 \\
\hline Síntesis & 2,38 \\
\hline Teoría de la Educación & 2,38 \\
\hline Universidad Internacional de La Rioja (UNIR) & 2,38 \\
\hline
\end{tabular}

El impacto que obtienen las publicaciones se mide en la actualidad por la categorización e indexación de las revistas y publicaciones en bases de datos o directorios de reconocido prestigio. La publicación sobre MOOC ha tenido una baja representación en revistas indexadas en bases de datos de alto impacto. La suma de artículos publicados en Social Science Ciation Index, Arts and Humanities Citation Index (ambas de Thomson Reuteurs) y SCOPUS (Scimiago) sólo representan el 8,93\% del total de artículos publicados. Las dos bases de datos con mayor representación son Dialnet y Latindex, ambas con el 8,93\% (Tabla 4). 
Tabla 4.

Porcentaje de publicación MOOC conforme a bases de datos y directorios

\begin{tabular}{lc}
\hline \multicolumn{1}{c}{ Bases de datos y directorios } & $\%$ \\
\hline Dialnet & 8,93 \\
Latindex & 8,93 \\
DOAJ & 8,67 \\
ISOC & 7,65 \\
CIRC & 5,36 \\
SCOPUS & 5,10 \\
RED ALyC & 4,85 \\
CARHUS PluS+ 2010 & 4,59 \\
ERA & 4,34 \\
SJR & 4,08 \\
FRANCIS & 3,83 \\
ACADEMIC SEARCH COMPLETE & 3,57 \\
CMMC & 3,57 \\
FUENTE ACADEMICA PREMIER & 3,57 \\
MLA & 3,57 \\
PAIS & 3,57 \\
SSCI & 3,57 \\
IRESIE & 1,79 \\
ERIC & 1,02 \\
ACADEMIC SEARCH PREMIER & 0,77 \\
SA & 0,77 \\
SIN INDEXACIÓN & 0,77 \\
SOCIAL SERVICES ABSTRACTS & 0,77 \\
SOCIOLOGICAL ABSTRACTS & 0,77 \\
WORLDWIDE POLITICAL SCIENCE ABSTRACTS & 0,77 \\
COMMUNICATION \& MASS MEDIA INDEX & 0,51 \\
FUENTE ACADEMICA & 0,51 \\
LINGUISTICS \& LANGUAGE BEHAVIOR ABSTRACTS & 0,51 \\
PSICODOC & 0,51 \\
SELLO DE CALIDAD FECYT & 0,51 \\
SPI & 0,51 \\
ABI/INFORM & 0,26 \\
AMERICAN HISTORY AND LIFE & 0,26 \\
ARTS AND HUMANITIES CITATION INDEX & 0,26 \\
HISTORICAL ABSTRACTS & 0,26 \\
INTERNATIONAL POLITICAL SCIENCE ABSTRACTS & 0,26 \\
LIBRARY AND INFORMATION SCIENCE ABSTRACTS & 0,26 \\
POLITICAL SCIENCE COMPLETE & 0,26 \\
\hline
\end{tabular}


Otro de los aspectos relevantes para la descripción y contextualización del movimiento MOOC a nivel nacional consiste en cuantificar la producción científica de los diferentes investigadores. En la Tabla 5 podemos comprobar el índice porcentual de la producción científica en MOOC de los autores que han publicado en revistas españolas con índice de impacto relativo. El total de autores con artículos MOOC en el intervalo analizado es de 89. Los dos primeros autores con filiación institucional en la Universidad de Huelva y el tercero en la Universidad Nacional de Educación a Distancia (UNED).

Tabla 5.

Autores por índice de publicación en $M O O C$

\begin{tabular}{lc}
\hline \multicolumn{1}{c}{ Autor } & $\%$ \\
\hline Aguaded, Ignacio & 3,33 \\
Medina-Salguero, Rosario & 2,22 \\
Vázquez-Cano, Esteban & 2,22 \\
Abuín Vences, Natalia; Acosta, E. S.; Alonso Arévalo, Julio; Alonso & \\
Berrocal, José Luis; Anglada, Lluís; Aranzadi, Pedro; Barbera, Elena; & \\
Bárcena, Elena; Betts, Alicia; Caballo, María Belén; Cabero Almena- & \\
ra, Julio; Cano García, Elena; Capdevila Pages, Ramón; Caride, José & \\
Antonio; Casañ, María José; Chauhan, Amit; Conole, Gráinne; Cooper- & \\
man, Larry; Cordón Gracía, José Antonio; Daza, V.; Díaz Flores, Sandro & \\
Raúl; Doherty, Kathleen; Escribano Otero, J. J.; Fernández, Alejandro; & \\
Filvà, Daniel Amo; Forment, Marc Alier; Gago Saldaña, David; García & \\
Aretio, Lorenzo; García Peñalvo, Francisco J.; Gasparini, Isabela; Gea & \\
Megías, Miguel; Gómez Díaz, Raquel; Gómez Galán, José; González, & \\
Héctor Matías; Gradaílle, Rita; Haywood, Jeff; Infante Moro, Alfonso; & \\
Kelder, Jo-Anne; King, Carolyn Elizabeth; Llorente Cejudo, María del & \\
Carmen; López Andrada, Concepción; López Meneses, Eloy; Luján- & \\
Mora, Sergio; Marauri Martínez de Rituerto, Pedro María; Marilza Per- & \\
nas, Ana, Martínez Abad, Fernando; Martín-Monje, Elena; McInerney, & \\
Fran; Méndez García, Carmen M.; Méndez Rey, Juan Manuel; Mengual & \\
Andrés, Santiago; Muñoz, Alfonso; Nadiu, Som; Palazzo Moreira de & \\
Oliveira, José; Pérez Ávila, Anabel; Pescador Albiach, Darío; Pose, Héc- & \\
tor M.; Ramón Aguirre, Jorge; Read, Timothy; Robinson, Andrew; Ro- & \\
dríguez Conde, María José; Roig Vila, Rosabel; Román Graván, Pedro; & \\
Rosana Montes Soldado; Salgado Labra, Isabel; Sancho, T.; Sangrá, & \\
Albert; Sarasola Sánchez-Serrano, José Luis; Silva Peña, Ilich; Sosa & \\
Díaz, M.a José; Suárez Guerrero, Cristobal; Torres Díaz, Juan Carlos; & \\
Torres Mancera, Daniel; Valdeni de Lima, José; Valderrama, F.; Valdi- & \\
viezo Díaz, Priscila; Valverde Berrocoso, Jesús; Vickers, James; Vinader & \\
Segura, Raquel; Walls, Justin; Wheeler, Steve; Wives, Leandro Krug; & \\
Zapata-Ros, Miguel. & \\
\hline & \\
\hline
\end{tabular}


Ninguno de los autores, debido a que el movimiento se encuentra todavía en fase de desarrollo, ha alcanzado la categoría de "gran productor» (10 o más trabajos) según la categorización de Quiles, Ortigosa, Pedroche y Méndez (2000) y sólo tres autores pueden considerarse como «aspirantes» (2-4 artículos o libros).

La representación de instituciones que han tenido un mayor índice de producción científica en MOOC han sido las universidades de Granada, Barcelona, Murcia y la Asociación Iberoamericana de Educación Superior a Distancia (AIESAD), que representan entre las cuatro el $64,28 \%$ del total de la producción científica analizada (Tabla 6).

Tabla 6.

Instituciones más representativas en la publicación MOOC

\begin{tabular}{lr}
\hline \multicolumn{1}{c}{ Nombre de la Institución } & $\%$ \\
\hline Universidad de Granada & 28,57 \\
Universidad de Barcelona & 14,29 \\
Asociación Iberoamericana de Educación Superior a Distancia (AIESAD) & 11,90 \\
Universidad de Murcia & 9,52 \\
Universitat Oberta de Catalunya & 7,14 \\
Grupo Comunicar & 4,76 \\
Universidad de Salamanca & 4,76 \\
Universidad Nacional de Educación a Distancia & 4,76 \\
Universidad Pablo de Olavide & 4,76 \\
Universidad Complutense de Madrid & 2,38 \\
Universidad de Santiago de Compostela & 2,38 \\
Universidad de Valencia & 2,38 \\
Universidad Internacional de La Rioja & 2,38 \\
\hline
\end{tabular}

El número de citas y el porcentaje que representan las publicaciones españolas conforme a su indexación en bases de datos de impacto proporciona un panorama muy revelador de su repercusión científica en el panorama de investigación en MOOC. La Tabla 7 muestra el número de citas y su porcentaje en dos de las bases de datos internacionales más prestigiosas en el mundo científico: Scopus y SSCI-WoS. 
Tabla 7.

Número de citas y porcentaje de las publicaciones MOOC en Scopus y SSCI

\begin{tabular}{|c|c|c|c|c|c|}
\hline \multicolumn{6}{|c|}{ Scopus } \\
\hline Carácter & 0 citas/ $\%$ & 1 citas/ $\%$ & 2 citas/ $\%$ & $30+\operatorname{citas} / \%$ & Sin indexar $/ \%$ \\
\hline Libros & $0 / 0,0$ & $0 / 0,0$ & $0 / 0,0$ & $0 / 0,0$ & $0 / 0,0$ \\
\hline Artículos & $14 / 36,8$ & $5 / 13,2$ & $0 / 0,0$ & $1 / 2,6$ & $18 / 47,4$ \\
\hline Total & $14 / 36,8$ & $5 / 13,2$ & $0 / 0,0$ & $1 / 2,6$ & $18 / 47,4$ \\
\hline \multicolumn{6}{|c|}{ Wos-SSCI } \\
\hline Carácter & 0 citas/ $\%$ & 1 citas/ $\%$ & 2 citas $/ \%$ & $30+\operatorname{citas} / \%$ & Sin indexar $/ \%$ \\
\hline Libros & $0 / 0,0$ & $0 / 0,0$ & $0 / 0,0$ & $0 / 0,0$ & $0 / 0,0$ \\
\hline Artículos & $2 / 5,3$ & $0 / 0,0$ & $0 / 0,0$ & $0 / 0,0$ & $36 / 94,7$ \\
\hline Total & $2 / 5,3$ & $0 / 0,0$ & $0 / 0,0$ & $0 / 0,0$ & $36 / 94,7$ \\
\hline
\end{tabular}

Los resultados muestran cómo el impacto por número de citas en Scopus y Wos-SSCI es significativamente bajo. Ningún artículo obtiene citas en revistas indexadas en SSCI. En Scopus seis artículos reciben al menos una cita, aunque con este resultado solo se alcanza una repercusión de cita en la base de datos ligeramente superior al 15\% del total de artículos publicados. Estos datos se han desagregado por años y comparado con bases de datos con menor impacto como «Google Scholar» (Tabla 8).

Tabla 8.

Porcentaje de citas en Google Scholar, Scopus y Wos-SSCI por años

\section{Citas recibidas}

\begin{tabular}{ccccc}
\hline Año & Número de citas & Google Scholar & Scopus & Wos/SSCI \\
\hline 2010 & 0 & 0,0 & 0,0 & 0,0 \\
2011 & 0 & 0,0 & 0,0 & 0,0 \\
2012 & 0 & 0,0 & 0,0 & 0,0 \\
2013 & $\mathbf{2 2}$ & 77,3 & 22,7 & 0,0 \\
2014 & $\mathbf{8}$ & 62,5 & 37,5 & 0,0 \\
Total & $\mathbf{3 0}$ & $\mathbf{7 3 , 3}$ & $\mathbf{2 6 , 7}$ & $\mathbf{0 , 0}$ \\
\hline
\end{tabular}

Los resultados de la Tabla 8 confirman que el impacto de la publicación de investigadores españoles en bases de reconocido prestigio es muy bajo. Las citas se concentran en los años 2013/14 y del total de 30 citas re- 
cibidas, solo un 26,7\% pertenecen a revistas indexadas en Scopus. El 73,3\% de las citas se obtienen de la base de datos Google Scholar en revistas con un menor índice de impacto.

Para complementar el impacto a nivel nacional hemos tomado en consideración la categorización ANEP/FECYT de las revistas que han publicado artículos sobre temática MOOC en el intervalo de tiempo estudiado (Tabla 9).

Tabla 9.

Publicación de artículos según categorización ANEP (2010-2014)

\begin{tabular}{crrrrrc}
\hline \multicolumn{7}{c}{ Categorización ANEP } \\
\hline Año & A+ & A & B & C & $\begin{array}{c}\text { Sin } \\
\text { categorizar } \\
\text { en ANEP }\end{array}$ & $\begin{array}{c}\text { Sin } \\
\text { categorizar } \\
\text { en DICE }\end{array}$ \\
\hline 2010 & 0,0 & 0,0 & 0,0 & 0,0 & 0,0 & 0,0 \\
2011 & 0,0 & 0,0 & 0,0 & 0,0 & 0,0 & 0,0 \\
2012 & 0,0 & 0,0 & 0,0 & 0,0 & 0,0 & 0,0 \\
2013 & 16,7 & 25,0 & 41,7 & 0,0 & 16,7 & 0,0 \\
2014 & 3,8 & 69,2 & 3,8 & 15,4 & 3,8 & 3,8 \\
Total & $\mathbf{7 , 9}$ & $\mathbf{5 5 , 3}$ & $\mathbf{1 5 , 8}$ & $\mathbf{1 0 , 5}$ & $\mathbf{7 , 9}$ & $\mathbf{2 , 6}$ \\
\hline
\end{tabular}

Los resultados según categorización ANEP/FECYT muestran un impacto relativamente alto. Los artículos publicados en categorización alta (A+ y A) alcanzan un 63,2\% del total de artículos publicados sobre temática MOOC y se concentran en los años 2013 y 2014.

Asimismo, otra de las bases de datos que hasta el año 2014 ha estado operativa y que ha servido de referencia para la medición del impacto de la publicación científica de las revistas españolas es In-Recs (http://ec3.ugr. es/in-recs/). En la Tabla 10, presentamos los resultados de la categorización según porcentaje y cuartil de impacto de los artículos publicados.

Tabla 10.

Porcentaje de publicación de artículos según categorización In-Recs

\begin{tabular}{cccccc}
\hline \multicolumn{7}{c}{ Cuartil In-Recs } \\
\hline Año & $\mathbf{1 .}^{\mathbf{0}}$ & $\mathbf{2 .}^{\mathbf{o}}$ & $\mathbf{3 .}^{\mathbf{0}}$ & $\mathbf{4 .}^{\mathbf{0}}$ & Sin Cuartil \\
\hline 2010 & 0,0 & 0,0 & 0,0 & 0,0 & 0,0 \\
2011 & 0,0 & 0,0 & 0,0 & 0,0 & 0,0
\end{tabular}




\begin{tabular}{crlrrr}
\hline \multicolumn{7}{c}{ Cuartil In-Recs } \\
\hline Año & $\mathbf{1 .}^{\mathbf{0}}$ & $\mathbf{2 .}^{\mathbf{0}}$ & $\mathbf{3 .}^{\mathbf{0}}$ & $\mathbf{4 .}^{\mathbf{0}}$ & Sin Cuartil \\
\hline 2012 & 0,0 & 0,0 & 0,0 & 0,0 & 0,0 \\
2013 & 25,0 & 8,3 & 8,3 & 25,0 & 33,3 \\
2014 & 46,2 & 0,0 & 23,1 & 7,7 & 23,1 \\
Total & $\mathbf{3 9 , 5}$ & $\mathbf{2 , 6}$ & $\mathbf{1 8 , 4}$ & $\mathbf{1 3 , 2}$ & $\mathbf{2 6 , 3}$ \\
\hline
\end{tabular}

Los resultados son moderadamente altos, ya que el 39,5\% de los artículos publicados pertenecen a revistas categorizadas en el primer cuartil de impacto.

Otro de los aspectos que mide el impacto de la producción científica es el número de revistas citantes, ya que constituye un indicador de diseminación e importancia de una temática. En la Tabla 11, se muestra el porcentaje y número de citas de las revistas citantes en MOOC.

Tabla 11.

Porcentaje y número de citas de las revistas citantes en MOOC

\begin{tabular}{lc}
\hline \multicolumn{1}{c}{ Nombre de la Publicación } & N / \% \\
\hline Sin cita & $28 / 51,85$ \\
Profesorado & $13 / 24,07$ \\
Digital Education Review & $3 / 5,56$ \\
Alabé & $1 / 1,85$ \\
Anuario Thinkepi & $1 / 1,85$ \\
@tic: Revista d'Innovació Educativa & $1 / 1,85$ \\
IJERI & $1 / 1,85$ \\
Open Praxis & $1 / 1,85$ \\
RED & $1 / 1,85$ \\
REDU: Revista de Docencia Universitaria & $1 / 1,85$ \\
Revista Nacional e Internacional de Educación Inclusiva & $1 / 1,85$ \\
RIED & $1 / 1,85$ \\
Vivat Academia & $1 / 1,85$ \\
\hline
\end{tabular}

La principal revista citante es "Profesorado" debido al monográfico 18(1) dedicado a la temática MOOC. El resto de la cita es relativamente bajo tanto en número de revistas como en las citas recibidas. La cita que se realiza suele ser de autores extranjeros en revistas internacionales, lo que minimiza la influencia del impacto de la publicación nacional en el índice de cita. 
Por último, uno de los aspectos que más incide en el impacto de la publicación sobre una temática es el carácter del enfoque metodológico que adoptan los artículos que se publican. En la Tabla 12, presentamos el porcentaje de artículos sobre temática MOOC según el enfoque metodológico.

Tabla 12.

Porcentaje del enfoque metodológico de los artículos MOOC

\begin{tabular}{cccccc}
\hline \multicolumn{5}{c}{ Enfoque metodológico } \\
\hline Año & Teóricos & Cuantitativos & Cualitativos & Mixtos & Otros \\
\hline 2010 & 0,0 & 0,0 & 0,0 & 0,0 & 0,0 \\
2011 & 0,0 & 0,0 & 0,0 & 0,0 & 0,0 \\
2012 & 0,0 & 0,0 & 0,0 & 0,0 & 0,0 \\
2013 & 38,5 & 7,1 & 7,1 & 28,6 & 14,3 \\
2014 & 51,7 & 20,7 & 0,0 & 20,7 & 6,9 \\
Total & $\mathbf{4 7 , 6}$ & $\mathbf{1 6 , 3}$ & $\mathbf{2 , 3}$ & $\mathbf{2 3 , 3}$ & $\mathbf{9 , 3}$ \\
\hline
\end{tabular}

Casi la mitad de los artículos publicados en el ámbito español son artículos teóricos $(47,6 \%)$. Este resultado es sintomático de la escasa repercusión internacional que está teniendo la publicación sobre MOOC en revistas españolas. La reflexión teórica es más dinámica en la red, muestra de ello es que autores de reconocido prestigio en el ámbito MOOC como Stephen Downes, George Siemens o Sir John Daniel publican sus reflexiones teóricas en entradas de blogs o en revistas de divulgación en línea y no en revistas de investigación. El ámbito de la investigación en MOOC es un campo que presenta una relativa dificultad de acceso a las plataformas para los investigadores independientes; lo que limita sin duda la publicación de estudios cuantitativos y cualitativos. Estas dificultades se pueden comprobar en el número de artículos de investigación con enfoque cuantitativo/cualitativo que se han publicado en el ámbito español que no alcanza el $20 \%$ del total.

\section{CONCLUSIONES}

Los resultados presentados en este artículo permiten dar cuenta del objetivo de esta investigación: cuantificar bibliométricamente la producción científica española con índice de impacto relativo en bases de datos de reconocido prestigio nacional e internacional sobre temática MOOC en el ámbito español y, por ende, proporcionar un panorama actualizado del estado de la cuestión en investigación científica sobre esta temática que sirva de referente para futuras investigaciones. 
Las variables presentadas en la investigación nos han permito analizar la evolución de la publicación de artículos y libros sobre MOOC en el intervalo (2010-2014) por área de conocimiento y comprobar cuáles han sido las principales revistas e instituciones con mayor índice de publicación en MOOC. Asimismo, hemos identificado los autores con publicaciones MOOC en el ámbito español y el porcentaje y número de citas que han recibido sus trabajos en bases de datos nacionales e internacionales (Wos-SSCI, Scopus, In-Recs, categorización ANEP/FECYT y Google Scholar). Estos resultados se han complementado con el porcentaje y número de citas de las revistas citantes en MOOC, las instituciones más representativas y el enfoque metodológico de los artículos MOOC.

Los resultados muestran que la repercusión de la temática MOOC en la comunidad científica española ha sido moderada. El área de conocimiento en que la temática MOOC ha tenido una mayor incidencia ha sido «Ciencias de la Educación» con un porcentaje del 88,10\% sobre el total de la producción científica en el campo de las Ciencias Sociales. Es significativo que no se haya publicado en el ámbito español ningún artículo en el inicio y gestación del movimiento MOOC (2010-2012). A partir de 2013, empieza la publicación tanto en artículos y libros y destaca que en la primera mitad del 2014 (27 artículos) se doble ya el número de artículos publicados en el 2013 (11 artículos). Cuatro revistas españolas acaparan el 54,75\% de las publicaciones sobre MOOC ("Profesorado. Revista de currículum y formación del profesorado", "RED. Revista de Educación a Distancia”, "Digital Education Review" y "RIED. Revista Iberoamericana de Educación a Distancia"). Los porcentajes más altos pertenecen a aquellas revistas que están empezando a realizar las «llamadas a artículos» sobre estas temáticas como la «Revista Profesorado» que alcanza un $26,19 \%$ de los artículos publicados motivado por la llamada a artículos que se realizó para el número 18(1).

El impacto de la publicación en MOOC en el ámbito español ha tenido una baja representación en revistas indexadas en bases de alto impacto. La suma de artículos publicados en Social Science Ciation Index, Arts and Humanities Citation Index y Scopus solo representan el 8,93\% del total de artículos publicados. Las dos bases de datos nacionales con mayor representación de artículos MOOC son Dialnet y Latindex, ambas con el 8,93\%.

Ninguno de los autores, debido a que el movimiento se encuentra todavía en fase de desarrollo, se le puede considerar un "gran productor" (10 o más trabajos) según la categorización de Quiles, Ortigosa, Pedroche y Méndez (2000). Esto implica que el número de citas internacionales es poco representativo entre los investigadores españoles. Las citas se concentran en los años 2013/14 y del total de 30 citas recibidas, solo un $26,7 \%$ pertenecen a revistas indexadas en Scopus. No existe ninguna cita en una re- 
vista indexada en Wos-SSCI. El impacto de las publicaciones MOOC según bases de datos nacionales es más acusado. Los artículos publicados según categorización ANEP/FECYT en (A+y A) alcanzan un 63,2\% y, conforme a los cuartiles de In-Recs, el 39,5\% de los artículos publicados pertenecen a revistas categorizadas en el primer cuartil de impacto.

Uno de los aspectos más significativos y, que puede explicar en buena medida la baja repercusión internacional, es que una gran parte de los artículos publicados $(47,6 \%)$ son artículos teóricos. La publicación de reflexiones teóricas tiene una versatilidad y periodicidad mayor en entradas en redes sociales, páginas web de investigadores y revistas de divulgación en la red. De hecho, los autores internacionales de reconocido prestigio en el mundo MOOC así lo atestiguan con constantes aportaciones en línea (entre otros: Stephen Downes, George Siemens o Sir John Daniel).

Por lo tanto, y derivado de este estudio, podemos concluir que, si bien el impacto educativo en España de los cursos MOOC es muy alto y así lo atestigua el informe de Oliver et al. (2014) para la Cátedra de Telefónica que posiciona a España entre las ocho potencias mundiales en este tipo de formación, el impacto científico internacional de las publicaciones españolas sobre MOOC es muy bajo y poco representativo en el intervalo de tiempo estudiado. 


\section{REFERENCIAS BIBLIOGRÁFICAS}

Aguaded, J. I. (2013). La revolución MOOCs, ¿una nueva educación desde el paradigma tecnológico? Comunicar, 41, 7-8.

Aguaded, J. I., Vázquez-Cano, E. y Sevillano, M. ${ }^{a}$ L. (2013). MOOCs, ¿turbocapitalismo de redes o altruismo educativo? En Scopeo Informe N. ${ }^{\circ} 2$ : MOOC: Estado de la situación actual, posibilidades, retos y futuro (pp. 7490). Salamanca: Universidad de Salamanca Servicio de Innovación y Producción Digital. Recuperado de http://scopeo.usal.es/wp

Alcaín Partearroyo, M. D. y San Millán Bujanda, M. J. (1993). Uso y tendencias de las técnicas bibliométricas en Ciencias Sociales y Humanas a nivel internacional. Revista Española de Documentación Científica, 16 (1), 30-41.

Anderson, T. (2013). Promise and/or Peril: MOOC and Open and Distance Education. Commonwealth of Learning. Recuperado de http://goo.gl/rY5BxV

Ariza, T., Granados, M. R., Ramiro, M. T. y Gómez-García, A. (2011). Una década de la Revista Española de Orientación y Psicopedagogía: un análisis bibliométrico de su evolución. Revista Española de Orientación y Psicopedagogía, 22, 38-57

Bartolomé, A.R. y Steffens, K. (2015). $¿$ Son los MOOC una alternativa de aprendizaje? Comunicar, 44, 91-99. http://dx.doi.org/10.3916/C44-2015-10

Buela-Casal, G. (2010). Índices de impacto de las revistas científicas e indicadores para medir el rendimiento de los investigadores. Revista de Psicodidáctica, 15, 3-19.
Cahlik, T. (2000). Comparisons of the Maps of Science. Scientometrics, 49, 373-387.

Chiu, W. T. y Ho, Y. S. (2005). Bibliometric analysis of homeopathy research during the period of 1991 to 2003. Scientometrics, 63(1), 3-23.

Christensen, G., Steinmetz, A., Alcorn, B., Bennett A., Woods, D. y Emanuel, E. J. (2013). The MOOC phenomenon: Who takes massive open online courses and why? Working Paper. University of Pennsylvania. Recuperado de http:// goo.gl/8QahHp

Conole, G. (2013). Designing for learning in an open world. New York: Springer.

Cormier, D. y Siemens, G. (2010). Through the open door: open courses as research, learning, and engagement. Educause Review, 45 (4), 30-39.

Daniel J. (2012). Making Sense of MOOCs: Musings in a Maze of Myth, Paradox and Possibility. Journal of Interactive Media in Education, 3. Recuperado de http://goo.gl/VZUHZm

DeBoer, J., Ho, A., Stump, G. y Breslow, L. (2014). Changing «course:» reconceptualizing educational variables for massive open online courses. Educational Researcher, 43 (2), 74-84.

Davis, J. C. y Gonzalez, J. G. (2003). Scholarly Journal Articles about the Asian Tiger Economies: Authors, Journals, and Research Fields, 1986-2001. AsianPacific Economic Literature, 17, 51-61.

Dillenbourg, P., Fox, A., Kirchner, C., Mitchell, J. y Wirsing, M. (Eds) (2014). Massive open online courses: current state and perspectives. Dagstuhl Manifestos. Schloss Dagstuhl - Leibniz Zentrum für Informatik. 
Downes, S. (2012). The Rise of MOOC. Recuperado de http://goo.gl/PL4G9l.

Downes, S. (2013). The Quality of Massive Open Online Courses. Recuperado de http://goo.gl/RqgVPd

Eaton, J. (2012). MOOC and Accreditation: Focus on the Quality of «Directto-Students». Education Council for Higher Education Accreditation, 9(1). Recuperado de http://goo.gl/WMjlun.

Estabrooks, C. A., Winther, C. y Derksen, L. (2004). Mapping the field: A bibliometric analysis of the research utilization literature in nursing. Nursing Research, 53, 293-303.

Fernández Cano, A. y Bueno Sánchez, A. (1998). Síntesis de estudios bibliométricos españoles en educación. Una dimensión evaluativa. Revista Española de Documentación Científica, 3 (21), 269-285.

Fernández Cano, A. (2011). Producción educativa española en el Social Sciences Citation Index (1998-2009). Revista Española de Pedagogía, 69 (250), 427-443.

Gómez-García, A., Ramiro, M. T., Ariza, T. y Granados, M. R. (2012). Estudio bibliométrico de Educación XX1. Educación XX1, 15, 17-41.

Guàrdia, L., Maina, M. y Sangrà, A. (2013). MOOC Design Principles. A Pedagogical Approach from the Learner's Perspective. eLearning Papers, 33. Recuperado de http://goo.gl/KBeSqR

Hoxby, C. M. (2014). The economics of online postsecondary education: MOOCs, nonselective education, and highly selective education. NBER Working Paper 19816. Recuperado de http://goo.gl/hyFvfc

Little, G. (2013). Massively Open? The Journal of Academic Librarianship, 39 (3), 308-309.
Neff, M. W. y Corley, E. A. (2009). 35 years and 160,000 articles: a bibliometric exploration of the evolution of ecology. Scientometrics, 80 (3), 657-682.

Oliver, M., Hernández-Leo, D., Daza, V., Martín, C. y Albó, L. (2014). Cuaderno: MOOCs en España. Cátedra TelefónicaUPF «Social Innovation in Education». Recuperado de http://goo.gl/UvDgHq

Pappano, L. (2012). Year of the MOOC. New York Times. Recuperado de http:// goo.gl/8ImbcE

Peritz, B. C. y Bar-Ilan, J. (2002). The sources used by bibliometrics scientometrics as reflected in references. Scientometrics, 54 (2), 269-284.

Quiles, M., Ortigosa, J., Pedroche, S. y Méndez, X. (2000). Investigaciones psicológicas sobre el cuidado del niño hospitalizado: Un análisis bibliométrico. Medicina Psicosomática y Psiquiatría de Enlace, 53, 27-42.

Radford, A. W., Robles, J., Cataylo, S., Horn, L., Thornton, J. y Whitfield, K. (2014). The employer potential of MOOCs: a survey of human resource professionals' thinking on MOOCs. RTI International. Recuperado de http://goo.gl/V409vr

Raposo, M., Martínez, E. \& Sarmiento, J.A. (2015). Un estudio sobre los componentes pedagógicos de los cursos online masivos. Comunicar, 44, 27-35. http://dx.doi.org/10.3916/C44-2015-03

Sangrà, A. (2013). Retos de los MOOCs. Recuperado de http://goo.gl/uWMbDx

Siemens, G. (2012). MOOCs for the win! ElearnSpace. Recuperado de http:// goo.gl/Ox8UFD

UNESCO (2013). Policy guidelines for mobile learning. Paris: United Nations Educational, Scientific and Cultural Organization. 
Vanti, N. (2000). Métodos cuantitativos de evaluación de la ciencia: Bibliometría, Cienciometría e Informetría. Investigación Bibliotecológica, 14 (29), 9-23.

Vázquez-Cano, E., López-Meneses, E. y Sarasola, J. L. (2013). La expansión del conocimiento en abierto: Los MOOCs. Barcelona: Octaedro.

Velasco, B., Eiros, J. M., Pinilla, J. M. y San Román, J. A. (2012). La utili- zación de indicadores bibliométricos para evaluar la actividad investigadora. Aula Abierta, 40, 75-84.

Yuan, L. y Powell, S. (2013). MOOCs and Open Education: Implications for Higher Education. Recuperado de http:// goo.gl/xCqo6q

Zapata, M. (2013). MOOC, una visión crítica. El valor no está en el ejemplar. Recuperado de http://goo.gl/JWkX14 


\section{ANEXO I \\ Artículos y libros sobre MOOC en revistas y editoriales españolas (2010-2014)}

\begin{tabular}{|c|c|c|c|c|}
\hline Título & $\begin{array}{l}\text { Libro / } \\
\text { Revista }\end{array}$ & $\begin{array}{l}\text { Revista / } \\
\text { Editorial }\end{array}$ & ISBN / ISSN & Año \\
\hline $\begin{array}{l}\text { Bases, mediaciones y futuro de la } \\
\text { educación a distancia en la socie- } \\
\text { dad digital }\end{array}$ & Libro & Síntesis & 9788499588148 & 2014 \\
\hline $\begin{array}{l}\text { El ecosistema del libro electróni- } \\
\text { co universitario }\end{array}$ & Libro & $\begin{array}{l}\text { Universidad de } \\
\text { Salamanca }\end{array}$ & 9788490122877 & 2014 \\
\hline $\begin{array}{l}\text { La expansión del conocimiento } \\
\text { en abierto: los MOOC }\end{array}$ & Libro & Octaedro & $978-84-9921-433-7$ & 2013 \\
\hline $\begin{array}{l}\text { Comunidades online, redes so- } \\
\text { ciales y redes de aprendizaje }\end{array}$ & Libro & UNIR & $978-84-15626-56-5$ & 2013 \\
\hline $\begin{array}{l}\text { Los MOOCs: viables, inquietan- } \\
\text { tes y consistentes }\end{array}$ & Artículo & Anu. Think EPI & $1886-6344$ & 2014 \\
\hline $\begin{array}{l}\text { Las tipologías de MOOC: Su di- } \\
\text { seño e implicaciones educativas }\end{array}$ & Artículo & Profesorado & $1138-414 \mathrm{X}$ & 2014 \\
\hline $\begin{array}{l}\text { Evaluación de la calidad pedagó- } \\
\text { gica de los MOOC }\end{array}$ & Artículo & Profesorado & $1138-414 X$ & 2014 \\
\hline $\begin{array}{l}\text { Los massive open on line courses } \\
\text { (MOOC) como extensión univer- } \\
\text { sitaria }\end{array}$ & Artículo & Profesorado & $1138-414 \mathrm{X}$ & 2014 \\
\hline $\begin{array}{l}\text { Los MOOC y la masificación per- } \\
\text { sonalizada }\end{array}$ & Artículo & Profesorado & $1138-414 \mathrm{X}$ & 2014 \\
\hline $\begin{array}{l}\text { El fenómeno MOOC y la univer- } \\
\text { salidad de la cultura: Las nuevas } \\
\text { fronteras de la Educación Supe- } \\
\text { rior }\end{array}$ & Artículo & Profesorado & $1138-414 X$ & 2014 \\
\hline $\begin{array}{l}\text { MOOC: Una visión crítica desde } \\
\text { las Ciencias de la Educación }\end{array}$ & Artículo & Profesorado & $1138-414 \mathrm{X}$ & 2014 \\
\hline $\begin{array}{l}\text { Experiencia de innovación edu- } \\
\text { cativa con curso MOOC: Los } \\
\text { códigos QR aplicados a la ense- } \\
\text { ñanza }\end{array}$ & Artículo & Profesorado & $1138-414 \mathrm{X}$ & 2014 \\
\hline $\begin{array}{l}\text { Los MOOC en la plataforma edu- } \\
\text { cativa MiriadaX }\end{array}$ & Artículo & Profesorado & $1138-414 X$ & 2014 \\
\hline $\begin{array}{l}\text { Utilización de MOOCS en la for- } \\
\text { mación docente: Ventajas, des- } \\
\text { ventajas y peligros }\end{array}$ & Artículo & Profesorado & $1138-414 \mathrm{X}$ & 2014 \\
\hline $\begin{array}{l}\text { La interacción entre compañeros } \\
\text { y el feedback lingüístico en los } \\
\text { COMA de lenguas extranjeras }\end{array}$ & Artículo & Profesorado & $1138-414 \mathrm{X}$ & 2014 \\
\hline
\end{tabular}




\begin{tabular}{|c|c|c|c|c|}
\hline Título & $\begin{array}{l}\text { Libro / } \\
\text { Revista }\end{array}$ & $\begin{array}{l}\text { Revista / } \\
\text { Editorial }\end{array}$ & ISBN / ISSN & Año \\
\hline $\begin{array}{l}\text { Evaluación del impacto del tér- } \\
\text { mino «MOOC» vs «ELearning»en } \\
\text { la literatura científica y de divul- } \\
\text { gación }\end{array}$ & Artículo & Profesorado & $1138-414 X$ & 2014 \\
\hline $\begin{array}{l}\text { Hacia una nueva interculturali- } \\
\text { dad (educativa) }\end{array}$ & Artículo & RED & $1578-7681$ & 2014 \\
\hline $\begin{array}{l}\text { Los moocs y su papel en la crea- } \\
\text { ción de comunidades de aprendi- } \\
\text { zaje y participación }\end{array}$ & Artículo & RIED & $1138-2783$ & 2014 \\
\hline $\begin{array}{l}\text { Figura de los facilitadores en los } \\
\text { Cursos Online Masivos y Abier- } \\
\text { tos (COMA / MOOC): nuevo rol } \\
\text { profesional para los entornos } \\
\text { educativos en abierto }\end{array}$ & Artículo & RIED & $1138-2784$ & 2014 \\
\hline $\begin{array}{l}\text { Los Cursos Online Masivos y } \\
\text { Abiertos: ¿oportunidad o ame- } \\
\text { naza para las universidades ibe- } \\
\text { roamericanas? }\end{array}$ & Artículo & RIED & $1138-2785$ & 2014 \\
\hline $\begin{array}{l}\text { Adaptatividade geocultural em } \\
\text { ambientes virtuais de aprendiza- } \\
\text { gem }\end{array}$ & Artículo & RIED & $1138-2786$ & 2014 \\
\hline $\begin{array}{l}\text { From elite to Mass to Universal } \\
\text { Higher Education: from distance } \\
\text { to open education }\end{array}$ & Artículo & RIED & $1138-2787$ & 2014 \\
\hline $\begin{array}{l}\text { Fit for Purpose': a cohort-centric } \\
\text { approach to MOOC design / Ade- } \\
\text { cuación al propósito»: un enfo- } \\
\text { que centrado en el colectivo de } \\
\text { estudiantes para el diseño de un } \\
\text { curso en línea masivo y abierto } \\
\text { (MOOC) }\end{array}$ & Artículo & RUSC & $1698-580 \mathrm{X}$ & 2014 \\
\hline $\begin{array}{l}\text { Approaches for quality in peda- } \\
\text { gogical and design fundamentals } \\
\text { in MOOCS }\end{array}$ & Artículo & $\begin{array}{l}\text { Teoría de la edu- } \\
\text { cación }\end{array}$ & $1138-9737$ & 2014 \\
\hline $\begin{array}{l}\text { La revolución MOOCs, ¿una nue- } \\
\text { va educación desde el paradigma } \\
\text { tecnológico? (editorial) }\end{array}$ & Artículo & Comunicar & $1134-3478$ & 2013 \\
\hline $\begin{array}{l}\text { El videoartículo: nuevo formato } \\
\text { de divulgación en revistas cientí- } \\
\text { ficas y su integración en MOOCs }\end{array}$ & Artículo & Comunicar & $1134-3478$ & 2013 \\
\hline $\begin{array}{l}\text { Nuevos modelos educativos: los } \\
\text { MOOCs como paradigma de la } \\
\text { formación online }\end{array}$ & Artículo & $\begin{array}{l}\text { Historia y Comu- } \\
\text { nicación Social }\end{array}$ & $1137-0734$ & 2013 \\
\hline
\end{tabular}




\begin{tabular}{|c|c|c|c|c|}
\hline Título & $\begin{array}{l}\text { Libro / } \\
\text { Revista }\end{array}$ & $\begin{array}{l}\text { Revista / } \\
\text { Editorial }\end{array}$ & ISBN / ISSN & Año \\
\hline $\begin{array}{l}\text { MOOCs as disruptive technolo- } \\
\text { gies: strategies for enhancing the } \\
\text { learner experience and quality of } \\
\text { MOOCs }\end{array}$ & Artículo & RED & $1578-7680$ & 2013 \\
\hline $\begin{array}{l}\text { Diseño e implementación de } \\
\text { cursos abiertos masivos en línea } \\
\text { (MOOC): expectativas y conside- } \\
\text { raciones prácticas }\end{array}$ & Artículo & RED & $1578-7680$ & 2013 \\
\hline $\begin{array}{l}\text { Nuevas formas de aprendizaje } \\
\text { informales: ¿O estamos formali- } \\
\text { zando lo informal? }\end{array}$ & Artículo & RUSC & $1698-580 X$ & 2014 \\
\hline $\begin{array}{l}\text { La ayuda pedagógica en los } \\
\text { MOOC's: un nuevo enfoque en la } \\
\text { acción tutorial }\end{array}$ & Artículo & @tic & $1989-3477$ & 2013 \\
\hline $\begin{array}{l}\text { Open learning recognition in tra- } \\
\text { ditional universities / Reconoci- } \\
\text { miento del aprendizaje abierto } \\
\text { en las universidades tradiciona- } \\
\text { les }\end{array}$ & Artículo & $\begin{array}{l}\text { Journal for Edu- } \\
\text { cators, Teachers } \\
\text { and Trainers }\end{array}$ & $1989-9572$ & 2013 \\
\hline $\begin{array}{l}\text { Bases per a un nou model de fi- } \\
\text { nançament de les universitats } \\
\text { públiques catalanes }\end{array}$ & Artículo & $\begin{array}{l}\text { Revista de Edu- } \\
\text { cación y Derecho }\end{array}$ & 2013-584X & 2013 \\
\hline $\begin{array}{l}\text { Retos en la financiación de la } \\
\text { educación superior }\end{array}$ & Artículo & $\begin{array}{l}\text { Revista de Edu- } \\
\text { cación y Derecho }\end{array}$ & $2013-584 X$ & 2013 \\
\hline $\begin{array}{l}\text { Comunidad de aprendizaje y } \\
\text { participación social en un curso } \\
\text { MOOC }\end{array}$ & Artículo & IJERI & $2386-4303$ & 2014 \\
\hline $\begin{array}{l}\text { Editorial: The Weakest Link As- } \\
\text { sessment and Accreditation in } \\
\text { MOOCs }\end{array}$ & Artículo & $\begin{array}{l}\text { Digital Educa- } \\
\text { tion Review }\end{array}$ & $2013-9144$ & 2014 \\
\hline $\begin{array}{l}\text { Massive Open Online Courses } \\
\text { (MOOCS): Emerging Trends in } \\
\text { Assessment and Accreditation }\end{array}$ & Artículo & $\begin{array}{l}\text { Digital Educa- } \\
\text { tion Review }\end{array}$ & 2013-9144 & 2014 \\
\hline $\begin{array}{l}\text { Motivación en la educación ma- } \\
\text { siva online. Desarrollo y experi- } \\
\text { mentación de un sistema de acre- } \\
\text { ditaciones para los MOOC }\end{array}$ & Artículo & $\begin{array}{l}\text { Digital Educa- } \\
\text { tion Review }\end{array}$ & 2013-9144 & 2014 \\
\hline $\begin{array}{l}\text { Estrategia para el seguimiento } \\
\text { y evaluación de los aprendizajes } \\
\text { en un MOOC de introducción al } \\
\text { álgebra }\end{array}$ & Artículo & $\begin{array}{l}\text { Digital Educa- } \\
\text { tion Review }\end{array}$ & $2013-9144$ & 2014 \\
\hline
\end{tabular}




\begin{tabular}{|c|c|c|c|c|}
\hline Título & $\begin{array}{l}\text { Libro / } \\
\text { Revista }\end{array}$ & $\begin{array}{l}\text { Revista / } \\
\text { Editorial }\end{array}$ & ISBN / ISSN & Año \\
\hline $\begin{array}{l}\text { Los Cursos en Línea Masivos y } \\
\text { Abiertos (MOOC) como alterna- } \\
\text { tiva para la educación a distancia }\end{array}$ & Artículo & GECONTEC & $2255-5684$ & 2014 \\
\hline $\begin{array}{l}\text { De la clase magistral al MOOC: } \\
\text { doce años de evolución de una } \\
\text { asignatura sobre la programa- } \\
\text { ción de aplicaciones web }\end{array}$ & Artículo & REDU & $1887-4592$ & 2013 \\
\hline $\begin{array}{l}\text { Crypt4you y la utilidad de los } \\
\text { MOOcs en la formación online } \\
\text { en lengua español }\end{array}$ & Artículo & $\begin{array}{l}\text { Innovación Edu- } \\
\text { cativa }\end{array}$ & $1130-8656$ & 2013 \\
\hline
\end{tabular}




\section{PERFIL ACADÉMICO Y PROFESIONAL DE AUTORES}

Dr. Ignacio Aguaded. Catedrático de Universidad del Departamento de Educación de la Universidad de Huelva (España). Presidente del Grupo Comunicar, colectivo veterano en España en Educomunicación, y Editor de la Revista Científica Iberoamericana "Comunicar» (indexada en JCR, Scopus, ERIH, RECYT...). Es además Director del Grupo de Investigación «Ágora», responsable de múltiples investigaciones nacionales e internacionales y la dirección de numerosas tesis doctorales. Es Director del Máster Internacional Interuniversitario de Comunicación y Educación Audiovisual (UNIA/ UHU) y Subdirector del Programa Interuniversitario de Comunicación (US, UMA, UCA y UHU). Ha sido Vicerrector de Tecnologías, Innovación y Calidad de la Universidad de Huelva durante 7 años (2005-12).

Dr. Esteban Vázquez-Cano. Profesor Acreditado a Titular de Universidad por la ANECA desempeña su labor docente e investigadora en el Departamento de Didáctica y Organización Escolar de la Facultad de Educación de la UNED. Sus líneas de investigación prioritarias son la Organización y Supervisión escolar, la Didáctica con TIC, el Aprendizaje móvil-ubicuo, el Lenguaje digital y los Cursos Online Masivos en Abierto (MOOC). Ha participado en diferentes proyectos de investigación nacionales e internacionales y en congresos de reconocido prestigio. Ha publicado más de 20 libros y 60 artículos en prestigiosas bases de datos (SSCI, SCOPUS, etc.). Es, asimismo, editor adjunto y miembro del comité científico de diferentes revistas científicas del área de Educación.

Dr. Eloy López-Meneses. Profesor Titular de Universidad en el Departamento de Educación y Psicología Social de la Universidad Pablo de Olavide (Sevilla, España). Director del Grupo de Investigación EduInnovagogía (HUM-971). Editor de la Revista «Internacional: «International Journal of Educational Research and Innovation» (IJERI). Dirección postal: Universidad Pablo de Olavide. Facultad de Ciencias Sociales. Especializado en Mooc, TIC y Diseño didáctico MEM. Doctor en Ciencias de la Educación y Premio extraordinario de tesis doctoral por la Universidad de Sevilla, Segundo premio Nacional en los Estudios de Ciencias de la Educación.

$\begin{array}{ll}\text { Dirección de los autores: } & \text { Ignacio Aguaded } \\ & \text { Universidad de Huelva } \\ & \text { Facultad de Educación } \\ & \text { Campus Universitario El Carmen } \\ & \text { 21071 Huelva } \\ & \text { E-mail: ignacio@aguaded.es }\end{array}$




\author{
Esteban Vázquez-Cano \\ Universidad Nacional de Educación a \\ Distancia \\ Facultad de Educación \\ C/ Juan del Rosal, 14 \\ 28040 Madrid \\ E-mail: evazquez@edu.uned.es \\ Eloy López-Meneses \\ Facultad de Ciencias Sociales \\ Universidad Pablo de Olavide \\ Ctra. de Utrera, km 1 \\ 41013 Sevilla. España \\ E-mail: elopmen@upo.es
}

Fecha Recepción del Artículo: 27. Septiembre. 2014

Fecha Aceptación del Artículo: 13. Febrero. 2015

Fecha Revisión para publicación: 11. Febrero. 2016 
